\section{CENTRO ESCOLAR FEDERAL EN BLUDENZ/AUSTRIA}

\author{
Manfred Nehrer y Reinhard Medek, \\ Arquitectos \\ $141-92$
}

\section{SINOPSIS}

Este centro escolar, considerado como uno de los más interesantes de Austria, se compone de cuatro cuerpos en los que se distribuyen, respectivamente, las escuelas, el gimnasio, la vivienda del conserje $y$ el local de transformadores.

En su construcción hay que destacar su adecuada flexibilidad, que permite dar cabida a las distintas funciones que en él se desarrollan, y la correcta adaptación del gran volumen edificado a las especiales características urbanas y arquitectónicas del ámbito circundante.
Este centro, que obtuvo el primer premio en un concurso de grupos escolares, está considerado en Austria como uno de los trabajos más interesantes en términos arquitectónicos.

Consta de un edificio principal, sala de gimnasio, vivienda para el conserje y central de transformadores. El cuerpo principal alberga, además del centro escolar propiamente dicho, una academia y una escuela de comercio, una escuela especial de profesiones femeninas y una escuela de hostelería.

Para este centro, los proyectistas tuvieron que resolver el problema de realizar el gran volumen exigido de manera' que la construcción se integrara armónicamente en el pequeño ámbito urbano de Bludenz. Esto se ha logrado mediante una adecuada estructuración en distintos niveles escalonados, consiguiendo que las imponentes masas constructivas del complejo escolar no aparezcan demasiado compactas o macizas desde ninguna orientación.

También en la realización de las fachadas hubo condicionantes especiales, debido a que todas las cosas de la vieja ciudad de Bludenz, incluidas las cercanas al nuevo complejo escolar presentan, por el estilo constructivo desarrollado en Vorarlberg

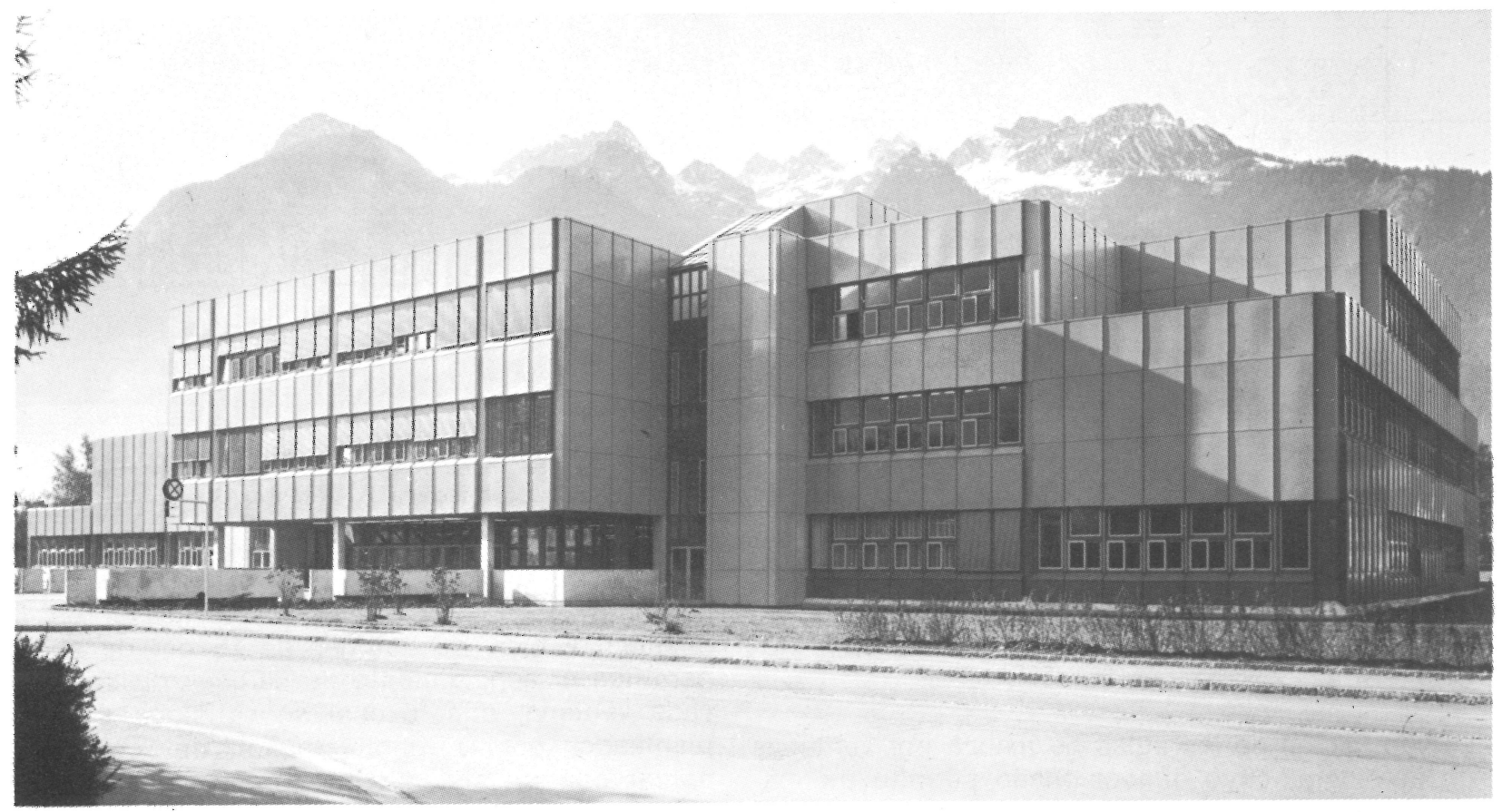


durante siglos, muy pequeñas superficies de ventanas subdivididas por listones. Este criterio tan humano, en cierta forma modificado, se ha aplicaco también en el nuevo centro. Asimismo se ha mantenido la tradición de pequeñas ventanas de corredera, tan frecuentes en las casas rústicas de Vorarlberg, así como se tuvieron en cuenta las condiciones especiales para el cumplimiento de las Disposiciones sobre aislamiento térmico, con el fin de procurar un ahorro en el consumo de energía. Estas disposiciones dictan que, para un edificio de estas características, las superficies acristaladas de las ventanas no debían sobrepasar el $40 \%$ del total de la superficie de fachada. En este caso los listones de las ventanas, particularmente los listones verticales de $15 \mathrm{~cm}$ de grueso dispuestos a distancias de $1,20 \mathrm{~m}$, están aislados $y$, por tanto, no computan en la superficie de ventanas ni gravan la economía térmica. Un detalle adicional sobre estas ventanas es que todas están diseñadas y dimensionadas de forma que pueden limplarse desde dentro.
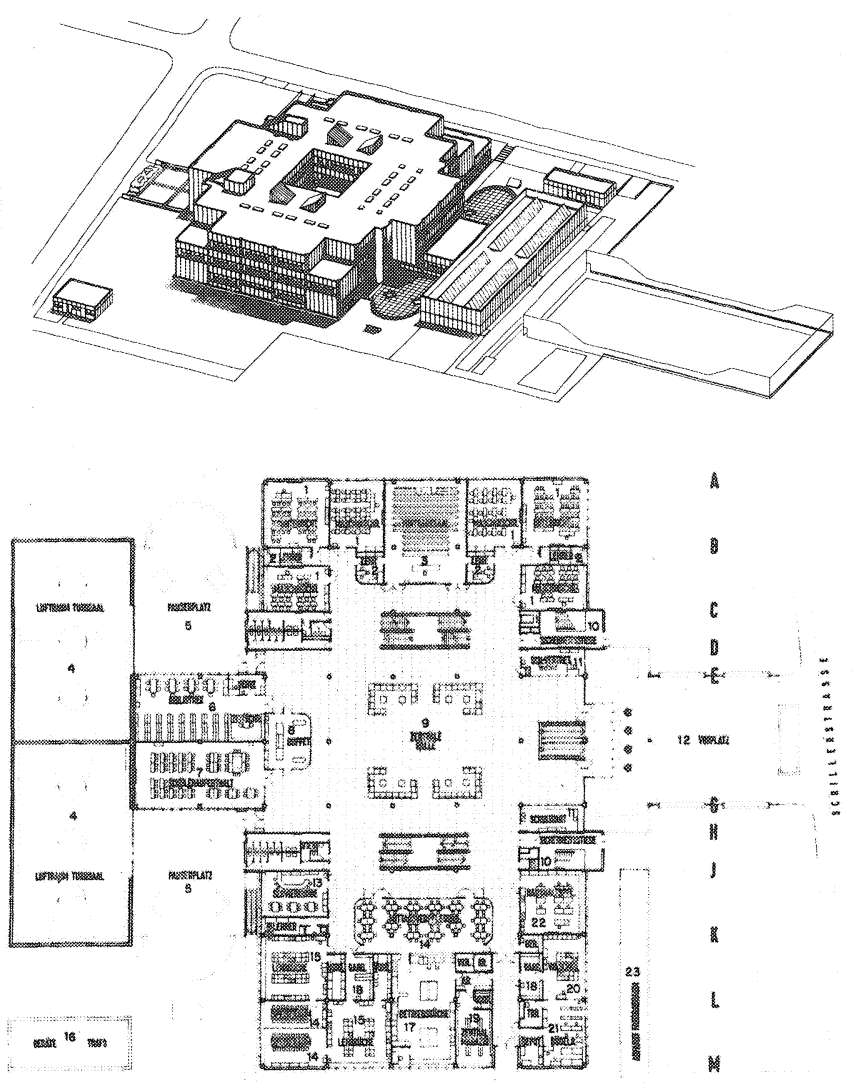

Planta baja

1. - Aula. 2. - Sala de profesores. 3.- Sala de conferencias. 4. Canchas de juego. 5. - Zona de recreo. 6. - Biblioteca. 7. - Sala de alumnos. 8. - Buffet. 9. - Hall principal. 10. - Escalera de emergencia. 11.-Conserje. 12.-Plaza de entrada. 13.-Bar. 14. Comedor. 15. - Cocina. 16. - Transformador. 17. -- Cocina central 18. - Guardarropa. 19. - Almacén central. 20. - Lavadero. 21. Planchador. 22. - Conservación. 23. - Salida garaje.

La ventilación de las aulas se realiza por ventanas de corredera, cuyo dimensionado permite un ma-

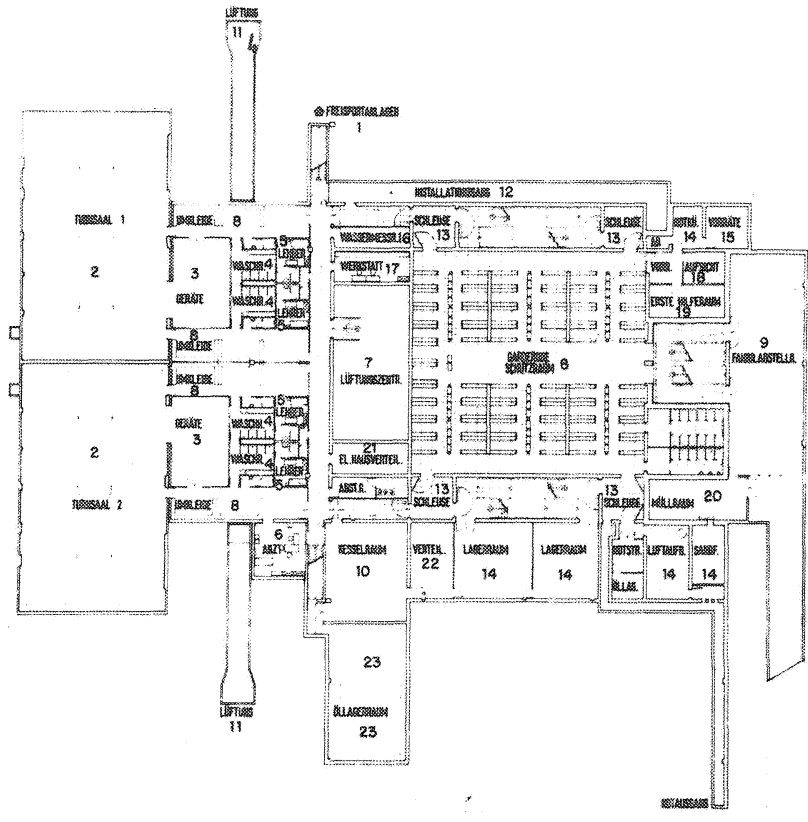

Primer sótano

1. Instalaciones al aire libre, 2.-. Canchas de juego. 3.-Aparatos. 4.-Duchas. 5.--Aseos (profesores). 6.-Botiquín. 7.Central de ventilación. 8. - Vestuarios. 9. --Bicicletas. 10.--Calderas. 11.--Ventilación. 12.--Pasillo de servicio. 13. - Cortavientos. 14.-Almacén. 15. - Cocina. 16. - Fontanería. 17.-Taller. 18.Vigilante. 19.-- Primeros auxilios. 20.--Basuras. 21.--Sala de distribución eléctrica. 22. - Distribución. 23.-Depósito de fuel-oil.

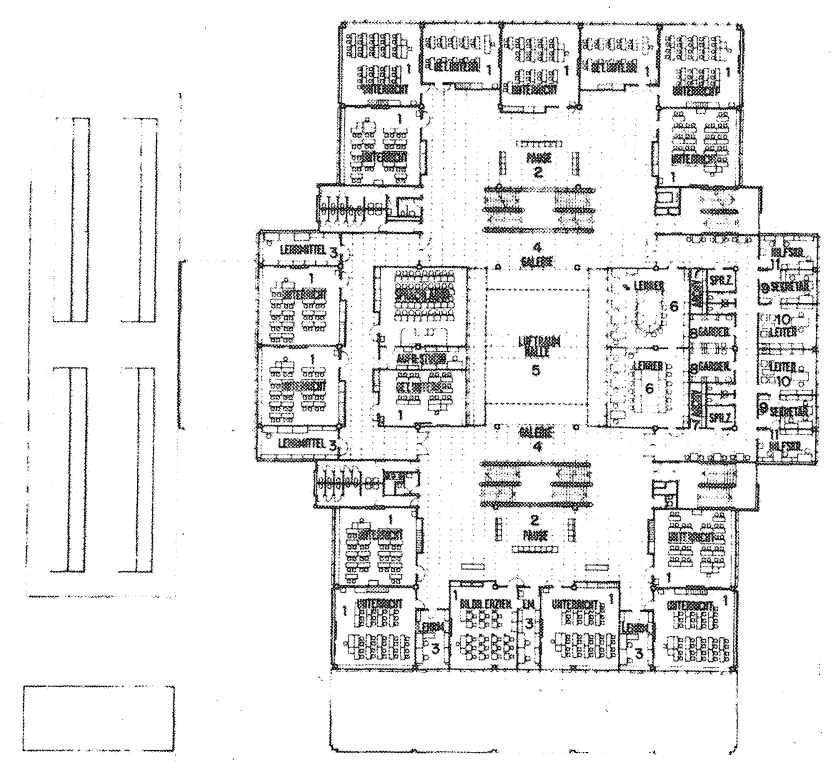

Planta primera

1.-Aula. 2.--Salón de recreo. 3.--Almacén. 4.-Galeria, 5.Patio. 6. - Sala de profesores. 7.--Archivo. 8.--Guardarropa. 9.Secretaría. 10.--Dirección. 11. --Ayudante.

nejo muy fácil. Las divisiones verticales de las mismas, a base de listones de $15 \mathrm{~cm}$ de ancho, permiten el acoplamiento de paredes divisorias en cada ventana, con lo cual se satisface el deseo planteado, por la administración del centro, de contar con un sistema que procurara la máxima 
flexibilidad posible para los tamaños de salas y clases.

Con el fin de animar las fachadas, han sido tratadas con diferentes tonos de color. El escalona. miento cromático se realizó mediante tonos maw rrones, más oscuros en la planta baja, y aclarándose progresivamente con la altura del edificio; de esta forma se obtuvo una disminución adicional del gran volumen construido.

Otro punto importante fue la distribución del espacio que, además de buscar una máxima funcionam lidad, intentaba exteriorizar las iunciones inte riores.

En el centro de la planta baja del cuerpo principal se ha dispuesto la gran sala de dos plantas de altura, que permite el contacto entre los alumnos de las distintas escuelas en los descansos entre clases. Esta sala está rodeada por el comedor - equipado con cocina para las comidas del me diodia-, la biblioteca, la sala de conferencias y los dos espacios de descanso. La gran sala, alumbrada desde arriba a través de un patio inte rior, constituye el centro del edificio y el ámbito común de las distintas escuelas. La cubierta de esta sala, a nivel de la $1 .{ }^{2}$ planta, está sostenida por una sofisticada construcción de acero, cuya configuración ha sido condicionada por la distribución de los espacios en dicha planta. Esta sim túa, en torno al patio, dos pequeños espacios de descanso y cuatro aulas, situadas unas y otras en lados opuestos del patio. Según esta disposición, la cubierta se sostiene mediante lucernarios que presentan distinta inclinación según sean los lados de las clases o los de las salas de descanso, y asi, mientras las primeras reciben luz y ventila. ción naturales a través del patio interior, las se gundas comunican especialmente con la gran sala, desarrollándose como galerias de la misma.

El segundo elemento de realización importante en la gran sala central son las dos dobles escaleras de tramos rectos, que se cruzan y enlazan en las mesetas intermedias, de forma que partiendo de un punto se puede llegar al mismo lado o viceversa.

En el ámbito de estas escaleras principales se hallan, en todas las plantas, los servicios y dos cajas de escaleras de seguridad protegidas contra el fuego, las cuales, en planta baja y en la superície del tejado, conducen al aire libre. En planta baja hay otras dos escaleras exteriores que, por una parte conducen al gimnasio, y por otra enlazan los vestuarios con las superficies deportivas exteriores.

Conviene mencionar el refugio creado en la planta sótano, con capacidad para 900 personas, y que normalmente se usa como vestuario o guardarro pa colectivo. Una entrada propia permite la util. zación extraescolar del complejo gimnástico.



Planta sagunda

1.-Aula. 2.-Salón de recreo. 3. - Sala de lectura. 4.-Seminario. 5. - Aula de Fisicoquímica. 6. - Aula técnica (oficina) 7. - Patio 8.-EDV. 9. - Sala de profesores. 10.--Aula de Ciencias Naturales. 11. - Sala de costura. 12.-Almacén.

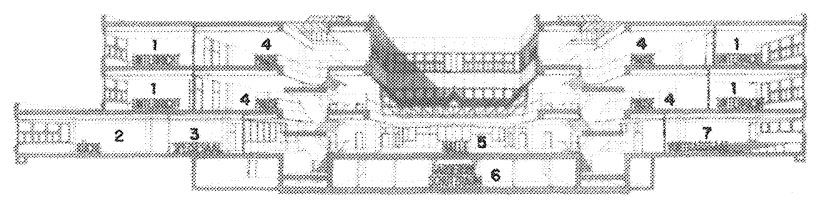

Sección-perspectiva

1.-Aula. 2.-Cocina. 3.-Comedor. 4.-Salón de recreo. 5.Hall. 6. - Vestuario. 7. - Sala de conferencias.

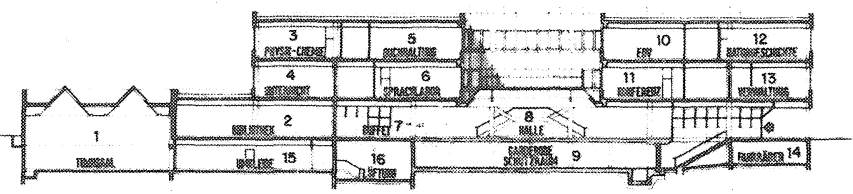

\section{Saccion}

1.-Gimnasio. 2.-Biblioteca. 3.-Aula de Fisicoquimica. 4.-Aula. 5.-Libreria. 6.-Laboratorio de idiomas. 7.-Buffet. 8. - Hall. 9. - Vestuario y guardarropa. 10. - EDV. 11. - Sala de conferencias. 12.-Aula de Ciencias Naturales. 13.-Administración. 14.-Bicicletas. 15. - Vestuario. 16. - Central de ventilación.

En cuanto a la distribución, se han reservado las zonas centrales para las dependencias que son de uso común por las distintas escuelas, situando las especificas en zonas más concretas. Asi, la planta 1. la ocupan esencialmente las secciones lingüisticas, mientras que la segunda contiene las 

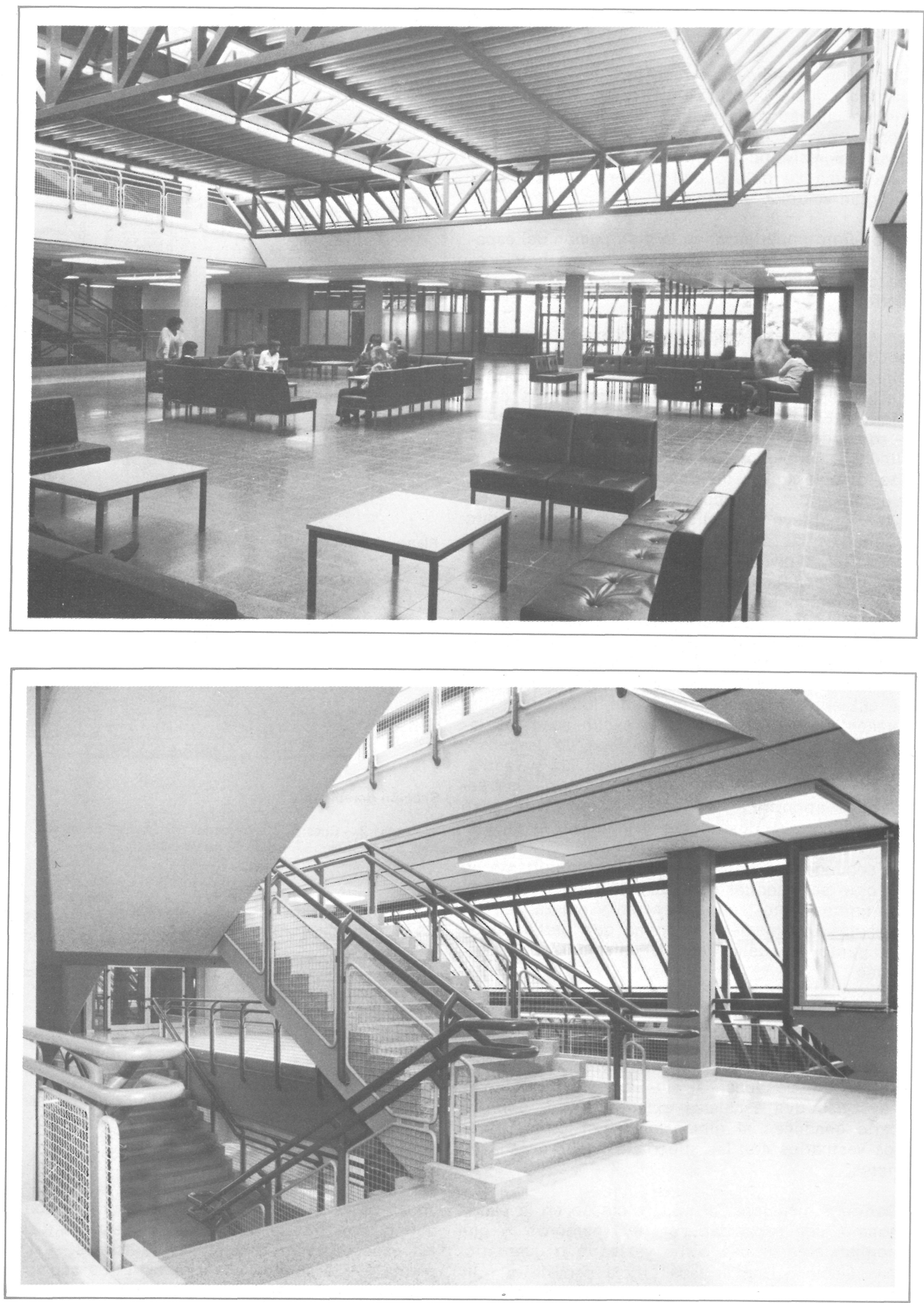


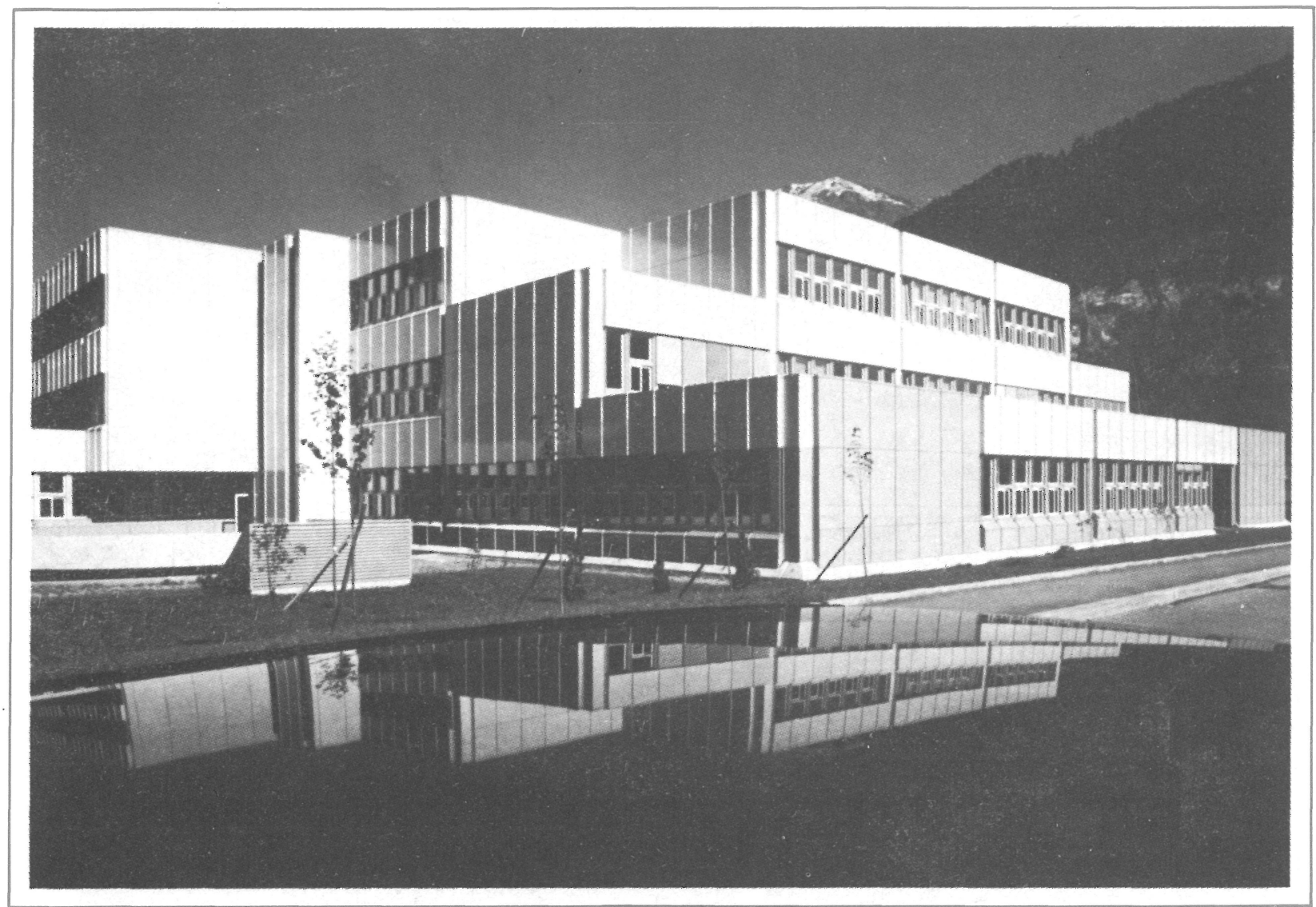

salas de ciencias naturales, matemáticas y las de profesiones femeninas. Cada uno de los cuatro ámbitos escolares de las plantas superiores se ha agrupado en torno a una pequeña sala de descanso.

El cuerpo del gimnasio y las terrazas exteriores de recreo separan el edificio escolar de las superficies deportivas al aire libre, evitándose molestias acústicas.

Para la construcción de los cimientos y de la planta sótano se empleó hormigón in situ, mientras que en las restantes plantas se utilizaron elementos estructurales prefabricados de gran formato $(8,40 \times 8,40 \mathrm{~m}=7 \times 1,20 \mathrm{~m}$-módulo europeo-).
Los muros ciegos exteriores, modulados con las ventanas, se compusieron con antepechos acumuladores de calor, a base de fábrica de hormigón de $30 \mathrm{~cm}$ de espesor con capa de $5 \mathrm{~cm}$ de aislate, y una fachada de chapa de aluminio con cámara de aire ventilada.

La tabiquería interior se hizo de construcción ligera, mediante módulos pequeños de 1,20 m de anchura. Las paredes divisorias entre las salas de clase se han constituido por dos tabiques de distinto espesor y una manta de lana de vidrio intermedia, para asegurar un eficiente aislamiento acústico. 